\title{
Conservation of mucosal associated invariant T (MAIT) cells and the MR1 restriction element in ruminants, and abundance of MAIT cells in spleen
}

\author{
Nick Goldfinch ${ }^{1}$, Peter Reinink ${ }^{2}$, Timothy Connelley ${ }^{1}$, Ad Koets ${ }^{2,3}$, \\ Ivan MORRISON $^{1}$, Ildiko VAN RHIJN ${ }^{2 *}$ \\ ${ }^{1}$ Infection and Immunity Division, The Roslin Institute, Royal (Dick) School of Veterinary Studies, \\ University of Edinburgh, Easter Bush, Roslin, Midlothian EH25 9RG, United Kingdom \\ ${ }^{2}$ Department of Infectious Diseases and Immunology, Faculty of Veterinary Medicine, Universiteit Utrecht, \\ Yalelaan 1, 3584CL, Utrecht, The Netherlands \\ ${ }^{3}$ Department of Farm Animal Health, Faculty of Veterinary Medicine, Universiteit Utrecht, Yalelaan 1, \\ 3584CL, Utrecht, The Netherlands
}

(Received 17 March 2010; accepted 27 May 2010)

\begin{abstract}
MHC-related protein 1 (MR1) is a highly conserved MHC class I-like molecule. Human and murine mucosal associated invariant T (MAIT) cells are restricted by MR1 and express an invariant T cell receptor. Even though MR1 protein expression on the cell surface has not been demonstrated in vivo or ex vivo, it is assumed that MR1 presents a bacterial antigen from the intestinal lumen to MAIT cells because MAIT cells are present in the lamina propria and their expansion is dependent on the presence of intestinal micro flora. The existence of bovine MAIT cells and MR1 has been demonstrated recently although ovine MAIT cells and MR1 have not yet been described. We cloned bovine and ovine MR1 transcripts, including splice variants, and identified an anti human MR1 antibody that recognizes cells transfected with the bovine homolog. Using this antibody, no MR1 staining was detected using cells freshly isolated from blood, thymus, spleen, colon, ileum, and lymph node. MAIT cells are known to be enriched in the CD4/CD8 double negative peripheral blood $\mathrm{T}$ cell population, but their relative abundance in different tissues is not known. Comparison of the amount of MAIT cell-specific TCR transcript to the amount of constant $\alpha$ chain transcript revealed that numbers of MAIT cells are low in neonates and increase by 3-weeks of age. In 3-month old animals, MAIT cells are abundant in spleen and less so in ileum, peripheral blood, lymph node, colon, and thymus.
\end{abstract}

MR1 / ruminant / T cell receptor / mucosal immunology / non-classical MHC

\section{INTRODUCTION}

MHC class I-like molecules form a group of proteins that share structural features, notably the characteristic MHC class I protein fold of the heavy chain and an ability to associate with $\beta_{2}$-microglobulin. The human MHC region on chromosome 6 encodes classical and non-

\footnotetext{
* Corresponding author: i.vanrhijn@uu.nl
}

classical MHC class I-like proteins, but other non-classical class I-like proteins such as human CD1 and MHC-related protein 1 (MR1) are located outside the $\mathrm{MHC}$ region $[3,14]$ at chromosome 1q23 and 1q25 respectively [15]. Comparison of human and murine MR1 sequences has shown that the $\alpha 1$ and $\alpha 2$ domains, which form the ligand binding part of MHC class I molecules, have the highest level of evolutionary conservation among all class 
I-related molecules [22]. However, the nature of the antigen bound to MR1 and how it is presented to T cells remain unclear. It has been suggested that, like CD1, MR1 presents lipid antigens $[12,16]$, but based on structural predictions others have suggested that hydrophilic antigens like proteins are more likely candidates $[5,6]$.

Although MR1 transcripts have been detected in all murine tissues examined, and in a range of human cell lines [14], cell surface expression of MR1 protein has not been detected in vivo or ex vivo [2, 5, 11, 24]. In transfected P388 and $\mathrm{L}$ cell lines, MR1 protein is produced, but is retained intracellularly $[11,24]$. However, human Hela cells and B6/WT-3 cells transduced with MR1 express the molecule at the cell surface $[5,11]$, where it exists in open and folded configurations that are suggestive of empty and antigen-bound forms respectively. MR1 protein produced in Escherichia coli fails to refold, possibly because of a lack of ligand [11]. Based on these observations, it has been suggested that cell surface expression of MR1 is tightly regulated in vivo, and possibly limited by the availability of ligand. Despite having a physical structure similar to MHC class I molecules, studies of MR1 intracellular transport indicate that, like CD1d, MR1 traffics through the endosomal pathway, where it is thought to acquire antigenic ligands [6].

Mucosal associated invariant T cells (MAIT cells) were initially described in humans, mice, and cattle based on the demonstration of a conserved TCR $\alpha$ chain rearrangement, expressing the orthologous TCR variable chain in each species and a highly conserved CDR3 sequence $[18,19]$. They represented a small subset of $\alpha \beta$ $\mathrm{T}$ cells that were enriched in the $\mathrm{CD} 4^{-} \mathrm{CD} 8^{-} \mathrm{T}$ cell population, but were also found in the CD8 ${ }^{\alpha \alpha+} \mathrm{T}$ cell population in humans, and in the murine $\mathrm{CD}^{+}$population [18]. In mice, development of MAIT cells has been shown to be dependent on MR1, $\beta 2 \mathrm{M}$, and an intact thymus, but independent of TAP, classical MHC molecules, and CD1. MAIT cell development has recently been shown to involve an initial intra-thymic selection, dependent on expression of MR1 and the presence of a haemopoietic-derived cell-type (not B-cells), giv- ing rise to low numbers of cells with a naïve T cell phenotype [9]. This is then followed by a phase of peripheral expansion, which is also dependent on MR1 and on B-cells and the presence of an intact gut flora, resulting in larger numbers of MAIT cells with a memory phenotype. Initial studies of tissue distribution in mice, based on measurement of MAIT TCR $\alpha$ transcripts, indicated that MAIT cells were most abundant in PBMC and lymph node, and were not present in the intestinal lamina propria or in the intra-epithelial lymphocytes (IEL) [18]. However, a later study demonstrated relatively large quantities of MAIT TCR transcripts in murine and human intestinal lamina propria, but not in IEL [19].

In addition to being a selecting element during T cell development, MR1 is recognized by some, but not all, MAIT cell hybridomas in vitro [19]. These and other findings indicate that MAIT cells recognize and respond to antigenic ligands presented by MR1 [2]. Recent work has demonstrated cross-reactivity of human and mouse MAIT cells with the respective MR1 orthologues, suggesting conservation of the ligands [7]. Upon activation, MAIT cells produce predominantly IL-4, IFN $\gamma$, and IL-17, and this triggers the release of pro-inflammatory cytokines from bystander cells, suggesting the ability of these cells to play a role in the regulation of inflammatory responses [17].

The current study set out to examine transcription and expression of MR1 and the distribution of MAIT cells in cattle and sheep and make a comparison with data obtained in humans and mice. The results confirmed that the MAIT-MR1 system is evolutionarily highly conserved among mammalian species and provided new evidence that MAIT cells are abundant in the spleen as well as gut-associated tissues.

\section{MATERIALS AND METHODS}

\subsection{Cloning and transfection of MR1}

Full-length bovine and ovine MR1 cDNA was cloned from PBMC using primers "boMR1for" 5'-GGGCTGATGATGCTCCTATTGCC-3' and 
"boMR1rev" 5'-GCAGCCATCTCCCATCAGTGT TC- $3^{\prime}$. Bovine PCR were performed with PFU Turbo polymerase (Stratagene, La Jolla, CA, USA) according to the protocol of the manufacturer under the following cycling conditions: an initial denaturation of $7 \mathrm{~min}$ at $95^{\circ} \mathrm{C}$, followed by 35 cycles of $30 \mathrm{~s}$ at $95{ }^{\circ} \mathrm{C}, 45 \mathrm{~s}$ at $60{ }^{\circ} \mathrm{C}, 1 \mathrm{~min}$ at $72{ }^{\circ} \mathrm{C}$, followed by a final elongation step of $7 \mathrm{~min}$ at $72{ }^{\circ} \mathrm{C}$. Ovine PCR used PCR master mix (ABgene, Epsom, UK) and DNA polymerase (Bioline BIOTAQ, London, UK), with cycling conditions: an initial denaturation of $10 \mathrm{~min}$ at $95^{\circ} \mathrm{C}$, followed by 35 cycles of $1 \mathrm{~min}$ at $95{ }^{\circ} \mathrm{C}, 1 \mathrm{~min}$ at $60{ }^{\circ} \mathrm{C}, 1 \mathrm{~min}$ at $72{ }^{\circ} \mathrm{C}$, followed by a final elongation step of $10 \mathrm{~min}$ at $72{ }^{\circ} \mathrm{C}$. PCR products were cut from an agarose gel, purified, and ligated in a Topo4blunt vector (Invitrogen, Breda, The Netherlands) (bovine) or pGEM-T Easy vector (Promega, Southhampton, UK) (ovine) for sequencing and in pcDNA3.1 for transient expression (bovine). Vector DNA of single colonies was sequenced by Baseclear (Leiden, The Netherlands) (bovine) or DBS Genomics (Durham University, UK) (ovine). For some experiments a second set of primers, "boovMR1f" 5'-CGGAGAGAGCAGTAC AAG-3" and "boovMR1r" 5'-CATATGGGACAGA GGAGG-3', was used to amplify full-length MR1; the PCR protocol using these primers was as described above, except with $\mathrm{Tm}=54{ }^{\circ} \mathrm{C}$.

\subsection{Antibodies and flow cytometry}

HEK 293 T cells were transiently transfected with bovine MR1 in pcDNA3.1 using Fugene- 6 reagent (Roche, Almere, The Netherlands) according to the manufacturer's protocol, and analysed $48 \mathrm{~h}$ after transfection. The following anti human MR1 antibodies were tested for reactivity against bovine MR1: clone 1 , clone 7 , clone 17 , clone 18 , clone 20 , clone 24 from the same hybridoma screen [5], kindly provided by Dr S. Gilfillan and Dr M. Cella (Department of Pathology and Immunology, Washington University School of Medicine, St. Louis, MO, USA), CC43, which recognizes an unidentified bovine class I-like molecule [4], and IL-A19, specific for classical bovine MHC class I. The anti-human MR1 antibody clone 20 was determined to be of the $\operatorname{IgG} 2 \mathrm{~b}$ isotype. For surface staining, cells were incubated for $30 \mathrm{~min}$ with unlabeled antibodies at $20 \mu \mathrm{g} / \mathrm{mL}$ in phosphate buffered saline containing $1 \%$ bovine serum albumin (Sigma-Aldrich, Zwijndrecht, The Netherlands) (PBS/BSA), followed by a wash and incubation for 30 min with phycoerythrin-labelled goat anti-mouse IgG2b (Southern Biotechnology Associates, Birmingham, AL, USA), FITC-labelled goat anti mouse IgG1 (BD Pharmingen, Breda, The Netherlands), or Alexa633-labelled goat anti-mouse Ig (Molecular Probes, Breda, The Netherlands). For staining of intracellular plus cell surface molecules, BD cytofix/cytoperm and BD perm/wash (BD Pharmingen) were used according to the supplied protocol. Anti-bovine CD3 MM1A (IgG1), antibovine CD21 gb25a (IgG1), and anti-bovine CD14 MM61A (IgG1) were obtained from VMRD (VMRD, Pullman, WA, USA).

\subsection{Sequence of the bovine and ovine MAIT cell TCR}

Primers (bovine) "I" 5'-GCTCTGCAGGAAAAG GCGTTAAG-3' and "K" $5^{\prime}$-TTTCAAAGCTTTTCTC TACCAGCTTGG-3', and (ovine) "boovUntr3f" 5-AG CGCCTGGTTTGTGGTGTC-3' and "CorovC3r" 5'-AGGTTAAACCCGACCACCTT-3', were applied to cDNA from bovine and ovine PBMC respectively, in PCR reactions using ABgene custom PCR mastermix and Bioline BIOTAQ DNA polymerase. Cycling conditions were an initial denaturation of $10 \mathrm{~min}$ at $95^{\circ} \mathrm{C}$, followed by 35 cycles of $1 \mathrm{~min}$ at $95^{\circ} \mathrm{C}$, $1 \mathrm{~min}$ at $60^{\circ} \mathrm{C}, 1 \mathrm{~min}$ at $72{ }^{\circ} \mathrm{C}$, followed by a final elongation step of $10 \mathrm{~min}$ at $72{ }^{\circ} \mathrm{C}$. PCR products were cloned into pGEM-T Easy vector and sequenced by DBS Genomics.

\subsection{Animals, tissue samples and cell lines}

Two 3-month old Holstein-Friesian bull calves (c1438 and c5524) were used to provide cells for flow cytometry and for extraction of nucleic acid from PBMC and tissue samples (prescapular lymph node, ileum, colon, spleen, thymus, and peripheral blood drawn from the jugular vein). Ovine tissue samples (prescapular lymph node, mesenteric lymph node, ileum, colon, spleen, thymus, and peripheral blood drawn from the jugular vein) were collected from two 6-month old sheep. Further samples of bovine tissues (prescapular lymph node, mesenteric lymph node, rumen wall, ileum, colon, spleen, thymus, and peripheral blood drawn from the jugular vein) were collected from additional HolsteinFriesian bull calves either within $24 \mathrm{~h}$ of birth (neonate), at 3-weeks of age, or 3-months of age. Calves kept to 3-weeks of age were also blood-sampled within 24-h of birth to allow animal-specific comparison of blood samples. At the time of tissue collection and blood sampling, these animals showed no clinical signs of disease. PBMC were harvested from blood using a standard Ficoll density gradient. Single cell suspensions of tissues from calves c1438 and 
c5524 were used without further purification. Ovine and other bovine tissues were homogenised using FastRNA ProGreen lysing matrix tubes (Mpbio, Hilton, UK) and QIAshredder tubes (Qiagen, Crawley, UK) to produce tissue lysate from which RNA was extracted.

Bovine cell lines representing different leukocyte lineages were derived by in vitro infection of bovine PBMC with either Theileria annulata or Theileria parva sporozoites as described previously [1]. The cell lines were phenotyped using flow cytometry as B-cell (592TPM, 663TA/B), macrophage (592TAA, 663TA), or T-cell (109TPM: CD8 +; 158TPM: mixed $\mathrm{CD} 8 / \gamma \delta$; 951TPM: $\gamma \delta)$.

\subsection{Cloning of MAIT TCR and qPCR for MAIT TCR $\alpha$ chain usage}

RNA isolation and cDNA synthesis was performed on $2 \times 10^{7}$ cells obtained from each tissue sample of calves c1438 and c5524. RNA was isolated with the Qiagen RNAEasy kit, followed by first strand cDNA synthesis with Multiscribe reverse transcriptase. RNA was isolated from ovine tissues and other bovine tissues using RNeasy Protect kit (Qiagen) and DNase-treated using Promega RQ1 RNase-free DNase. cDNA synthesis was performed using AMV reverse transcriptase (Promega). To generate a construct that could be used as a positive control and to generate standard curved for qPCR, a bovine primer set "I"/ " $\mathrm{K}$ " and an ovine pimer set "boovUntr3f"/"CorovC3r", designed to anneal to the $\mathrm{V}$ segments that are used by bovine and ovine MAIT cells and the respective $\alpha$ constant chains, were used to create $750 \mathrm{bp}$ PCR products. These were cloned and sequenced to confirm MAIT TCR $\alpha$ chain.

For the MAIT-cell V-region-specific qPCR the following primers/probe were used - Forward: "boAV19" 5'-CATTCCTTAGACGCTCTGATGCA CA-3' [18]; Reverse (bovine): "boAJ33" 5'-GCCCCA GATCCACTGATAGTTGC-3' [18]; Reverse (ovine): "ovAJ33" 5'-GCCCCAGATCAACCGATAGTTG C-3'; Probe: "boAV19" FAM-5'-TTACCTCCTTC TGAAGGAACTCCACATGAAAGA-3'-BHQ. For the $\alpha$ chain constant-region-specific qPCR the following primers/probe were used (1) for bovines c1438 and c5524 - Forward: "alpha" 5'-GATGC GAATACACCTTCAACG-3'; Reverse: “K” 5'-TT TCAAAGCTTTTCTCTACCAGCTTGG-3'; Probe: "const alpha" FAM-5'-TCCCTTCGCCTCCAGCT TGGAAATCT-3'-BHQ; (2) for ovine and other bovine tissues - Forward: "NGconst-f" 5 -ACGGGA TAGTGACTTGGGGAA-3'; Reverse: "NGconst-r"
5'-TTTCAAAGCTTTTCTCTACCAGCTTGG-3'; Probe: "NGconst" FAM-5'-ACACCTTCAACGAGAACA-3'. The location of the primers is shown in Supplementary Figure 1 available online at www.vetres.org.

On cDNA from bovines c1438 and c5524, qPCR were performed in a $25 \mu \mathrm{L}$ reaction volume containing $600 \mathrm{nM}$ of each primer, $100 \mathrm{nM}$ of the probe, and 10 ng of cDNA in TaqMan Universal PCR Master Mix (Applied Biosystems, Nieuwerkerk a/d IJssel, The Netherlands). Ovine and other bovine qPCR were performed in a $20 \mu \mathrm{L}$ reaction volume containing $500 \mathrm{nM}$ of each primer, $250 \mathrm{nM}$ of probe, and $3 \mu \mathrm{g}$ of cDNA in Taqman Gene Expression Master Mix (Applied Biosystems). Both reactions used the cycling conditions: an initial incubation of $2 \mathrm{~min}$ at $50{ }^{\circ} \mathrm{C}$, an incubation for $10 \mathrm{~min}$ at $95^{\circ} \mathrm{C}$, and 50 cycles of $10 \mathrm{~s}$ (ovine $15 \mathrm{~s}$ ) at $95{ }^{\circ} \mathrm{C}$ and $1 \mathrm{~min}$ at $53{ }^{\circ} \mathrm{C}$.

\subsection{Database searches and alignments}

A BLAST search was performed in the bovine genome $^{1}$ using the nucleic acid sequence of the $\alpha 1$ and $\alpha 2$ domains of human MR1. The obtained sequence was used for a BLAST search in the NCBI databases $^{2}$ to identify ovine and bovine MR1 transcripts in the EST databases. The Translate Nucleic Acid Sequence Tool was used ${ }^{3}$ for translation into amino acids. All alignments and phylogenetic trees were generated with ClustalW ${ }^{4}$.

\subsection{Statistical analyses of data}

Real-time PCR results were assessed using general linear mixed effect models using $\mathrm{R}$ version 2.9, a programming language and software environment for statistical computing, available from http:// www.r-project.org.

\section{RESULTS}

\subsection{Cloning of bovine and ovine MR1 transcripts}

The bovine MR1 gene ENSBTAG0000000 9924 on chromosome 16 is available at www. ensembl.org/Bos_taurus, version Btau_4.0.

\footnotetext{
${ }^{1}$ www.ensembl.org/Bos taurus, version 3.1, release 45 , www.ensembl.org

${ }^{2}$ www.ncbi.nlm.nih.gov/BLAST

3 http://biotools.umassmed.edu

${ }^{4}$ http://align.genome.jp
} 
Using PCR, we obtained a full-length transcript from bovine PBMC cDNA, but our full-length MR1 sequence had different leader and cytoplasmic tail sequences from the predicted Ensembl transcript. The leader and cytoplasmic tail sequences that we cloned were supported by (partial) EST sequences, suggesting that the Ensembl predicted splice sites were incorrect.

Full-length ovine MR1 cDNA was obtained as a clone from a PCR product obtained using primers designed from partial bovine MR1 EST sequences applied to ovine PBMC cDNA. The full length bovine and ovine MR1 cDNA sequences that we obtained are available at www.ncbi.nlm.nih.gov under accession numbers FJ028657 and FJ423039 and shown in Supplementary Figure 2.

The intron/exon structure and the nucleotide sequences of bovine and ovine full-length MR1 transcripts show a high level of similarity to murine and human full-length " $A$ " transcripts [14]. Like in humans and mice [14, 23], the ruminant genes have unusually long introns. The $\alpha 1$ and $\alpha 2$ domains of bovine MR1 show $81 \%$ and $85 \%$ identity to murine and human MR1 respectively at the amino acid level (Fig. 1); the murine and human sequences are $90 \%$ identical. This compares with $73 \%$ identity for the corresponding domains of bovine and human CD1b, and $61 \%$ for murine and human CD1d. A detailed comparison of individual domains of the bovine and ovine MR1 sequences is given in Table I.

\subsection{Alternatively spliced variants of MR1 in cattle and sheep}

Analysis of PCR products obtained from bovine and ovine PBMC using primers designed to generate full-length sequences of MR1 revealed a prominent band of approximately $900 \mathrm{bp}$ in addition to the band of the predicted size of the full length product of approximately $1100 \mathrm{bp}$. An additional band of about $550 \mathrm{bp}$ was detected in the bovine PCR product (Fig. 2A, upper panel). Sequencing of these products demonstrated that they represent splice variants, named boMR1.900,
boMR1.550, and ovMR1.900 (accession numbers FJ423041, FJ423042, FJ423040), from which either the complete $\alpha 1$ (boMR1.900, ovMR1.900), or $\alpha 1$ and $\alpha 3$ (boMR1.550) exons are missing (Fig. 2B). These sequences did not contain internal stop codons and retained the original reading frame. Hence, they potentially can generate truncated forms of the MR1 protein. Application of this PCR to cDNA from a variety of Theileria spp.-immortalized cell-lines of $\mathrm{T}$ and $\mathrm{B}$ lymphocyte lineage and monocyte lineage demonstrated that the $1100 \mathrm{bp}$ full length transcript and the $900 \mathrm{bp}$ splice variant were both abundant in all of the lines (Fig. 2A, lower panel).

\subsection{MR1 protein expression is not detectable in vivo as determined by flow cytometry}

Full-length bovine MR1 cDNA was cloned into pcDNA3.1 and used for transient transfection of HEK 293 T cells, COS-7 cells, and the bovine macrophage cell line Bomac. A panel of monoclonal antibodies specific for human MR1 was tested for reactivity against bovine MR1. Only the anti-human MR1 antibody clone 20 recognized bovine MR1. Using a protocol that stains intracellular plus cell surface molecules, a low percentage of cells positively staining with clone 20 was observed among the transfected cells (Figs. 3A-3C). No cell surface expression of bovine MR1 could be detected on the transfected cells (data not shown). Additional monoclonal antibodies specific for classical bovine MHC class I (IL-A19), bovine CD1 (20.27 and SBUT6) [8, 21] and an unidentified bovine class I-like molecule (CC43) $[4,21]$ were tested for cross-reactivity with bovine MR1 using the protocol for intracellular plus cell surface staining, and did not stain bovine MR1 (data not shown).

Flow cytometric analyses were performed on freshly isolated bovine PBMC, and cell suspensions obtained from thymus, lymph node, ileum and colon (data not shown), and spleen (Figs. 3D-3F). Using the anti-human MR1 antibody clone 20 followed by Alexa633-labelled goat anti-mouse in a staining protocol for intracellular plus cell surface molecules, no MR1 expression was detected in spleen. As a positive 


\begin{tabular}{|c|c|}
\hline \multirow{4}{*}{$\begin{array}{l}\text { boMR1 } \\
\text { ovMR1 } \\
\text { huMR1 } \\
\text { muMR1 }\end{array}$} & \begin{tabular}{l|l} 
Leader & Extracellular domain \\
\cline { 2 - 2 } RTHSLRYFRLGISEPGYGIPFFISAGYVDSHPITMYNS
\end{tabular} \\
\hline & MMLLLPLI IVLMMKLSDARTHSLRYFRLGISEPGYGIPEFISAGYVDSHPITMYNS \\
\hline & MGELMAFLLPLIIVLMVKHSDSRTHSLRYFRLGVSDPIHGVPEFISVGYVDSHPITTYDS \\
\hline & MMLLLPLLAVFLVKRSHTRTHSLRYFRLAVSDPGPVVPEFISVGYVDSHPITTYDS \\
\hline & 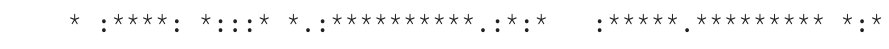 \\
\hline OMR1 & VSQLKEPRALWMEENLAPDHWERYTQLLRGWQQAFKVELKQLQHHYNHSGFHTYQRMIGC \\
\hline vMR1 & VSQLKEPRAPWMAENLEPDHWERYTQLLRGWQQAFKVELKQLQHHYNHSGFNTYQRMIGC \\
\hline & VTRQKEPRAPWMAENLAPDHWERYTQLLRGWQQMFKVELKRLQRHYNHSGSHTYQRMIGC \\
\hline & VTRQKEPKAPWMAENLAPDHWERYTQLLRGWQQTFKAELRHLQRHYNHSGLHTYQRMIGC \\
\hline & 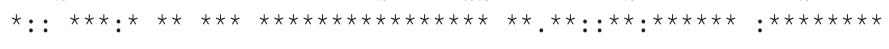 \\
\hline OMR1 & ELLEDGSITGFLQYAYDGQDFLIFNKDTLSWMAMDNVADI IRRVWEANRHELQYQKNWLE \\
\hline VMR1 & ELLDDGSTTGFLQYAYDGQDFI IFNKDTLSWIAMDNVANI IRRAWEANRHELQYQKNWLE \\
\hline MR1 & ELLEDGSTTGFLQYAYDGQDFLIFNKDTLSWLAVDNVAHTIKQAWEANQHELLYQKNWLE \\
\hline & ELLEDGSTTGFLQYAYDGQDFI IFNKDTLSWLAMDYVAHITKQAWEANLHELQYQKNWLE \\
\hline & 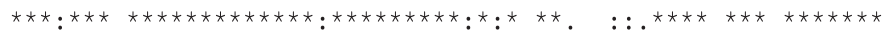 \\
\hline OMR1 & EECIAWLKRFLEYGKDALQRTEPPKVRVNHKETFPGITTLYCRAYGFYPPEISINWMKNG \\
\hline & EECIAWLKRFLEYGKDTLQRTEPPKVRVNYKETEPGITTLYCRAHGFYPPEISINWMKNG \\
\hline MR1 & EECIAWLKRFLEYGKDTLQRTEPPLVRVNRKETFPGVTALFCKAHGFYPPEIYMTWMKNG \\
\hline P 1 & EECIAWLKRFLEYGRDTLERTEHPVVRTTRKETFPGITTFFCRAHGFYPPEISMTWMKNG \\
\hline & 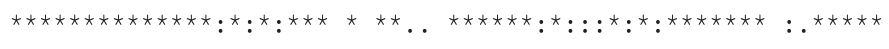 \\
\hline MR1 & EEIFQDTDYGGILPSGDGTYQTWVSVELDPQNGDIYSCHVEHGGVHMVLQGFQESETIL \\
\hline vMR1 & EEVVQDTNYGGILPSGDGTYQTWVSVELDSQNGDIYSCHVEHGGVHMVLPGFQDSETML \\
\hline & EEIVQEIDYGDILPSGDGTYQAWAS IELDPQSSNLYSCHVEHCGVHMVLQVPQESETIP \\
\hline & EEIAQEVDYGGVLPSGDGTYQTWLSVNLDPQSNDVYSCHVEHCGRQMVLEAPRESGDIL \\
\hline & 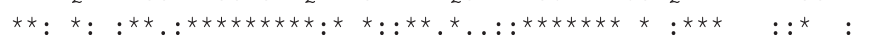 \\
\hline & Transmembrane $\quad$ Cytoplasmic domain \\
\hline oMR1 & |LVVKAVG-FIVLAIALAGVGILA|WRKRPRGKNKVICLSTPEH \\
\hline & LVMKAVG-FIVLAIALAGVGILAWRRKPRGENKVIYLSTPEH \\
\hline & LVMKAVSGSIVLVIVLAGVGVLVWRRRPREQNGAIYLPTPDR \\
\hline & RVSTISG-TTILIIALAGVGVLIWRRSQELKEVMYQPTQVNEGSSPS \\
\hline & $\star . \quad . \quad: * \star * \star \star * \star * * * \star: \quad .::: \quad . \quad:$ \\
\hline
\end{tabular}

Figure 1. Alignment of MR1 sequences from different species. Alignment of full-length bovine (bo) (accession number FJ028657.1), ovine (ov) (FJ423039), human (hu) (AJ249778.1), and murine (mu) (AF010448.1) MR1. $(*)$ Indicates a position with a fully conserved residue, (:) indicates strong conservation, and (.) indicates weak conservation.

control for the staining procedure, an antibody against bovine $\mathrm{CD} 3$ was used. In separate experiments, PBMC were double stained with anti-human MR1 antibody clone 20 in combination with antibodies against CD3, CD14, and CD21 using a protocol that stains intracellular plus cell surface molecules (Figs. 3G-3I). No expression of MR1 could be detected in any of the cell populations, despite detection of specific staining of MR1-transfected cells using the same staining method (Figs. 3A-3C). It should be noted however that the sensitivity of the staining may not have been sufficient to detect very low levels of MR1 expression.

\subsection{Sequence of the bovine and ovine MAIT cell TCR}

Primer I, specific for the V segment that is used by bovine MAIT cells $[13,18]$ and primer $\mathrm{K}$, specific for the $\alpha$ constant gene segment, respectively, were used to generate a full-length TCR $\alpha$ chain PCR product from cDNA prepared 
Table I. Cross-species comparison of MR1 nucleotide sequence. Comparison of ovine (Ov), bovine (Bo), murine $(\mathrm{Mu})$, and human $(\mathrm{Hu}) \mathrm{MR} 1$ sequences show a high percentage identity when $\alpha 1, \alpha 2$ and $\alpha 3$ domains are compared across species.

\begin{tabular}{lcccccc}
\hline $\begin{array}{l}\text { Sequences } \\
\text { compared }\end{array}$ & $\begin{array}{c}\text { Leader } \\
(55 \mathrm{bp}) \\
\text { in \% }\end{array}$ & $\begin{array}{c}\alpha 1 \\
(261 \mathrm{bp}) \\
\text { in \% }\end{array}$ & $\begin{array}{c}\alpha 2 \\
(276 \mathrm{bp}) \\
\text { in \% }\end{array}$ & $\begin{array}{c}\alpha 3 \\
(276 \mathrm{bp}) \\
\text { in \% }\end{array}$ & $\begin{array}{c}\text { TM } \\
(<105 \mathrm{bp}) \\
\text { in \% }\end{array}$ & $\begin{array}{c}\text { Cyt } \\
(>40 \mathrm{bp}) \\
\text { in \% }\end{array}$ \\
\hline Ov:Bo & 100 & 97 & 95 & 96 & 94 & 95 \\
Ov: $\mathrm{Mu}$ & 76 & 79 & 86 & 75 & 58 & 55 \\
Ov: $\mathrm{Hu}$ & 82 & 84 & 88 & 83 & 79 & 75 \\
Bo: $\mathrm{Mu}$ & 76 & 78 & 86 & 76 & 58 & 58 \\
Bo: $\mathrm{Hu}$ & 82 & 83 & 88 & 84 & 80 & 73 \\
Hu:Mu & 73 & 86 & 87 & 82 & 64 & 65 \\
\hline
\end{tabular}

(a)
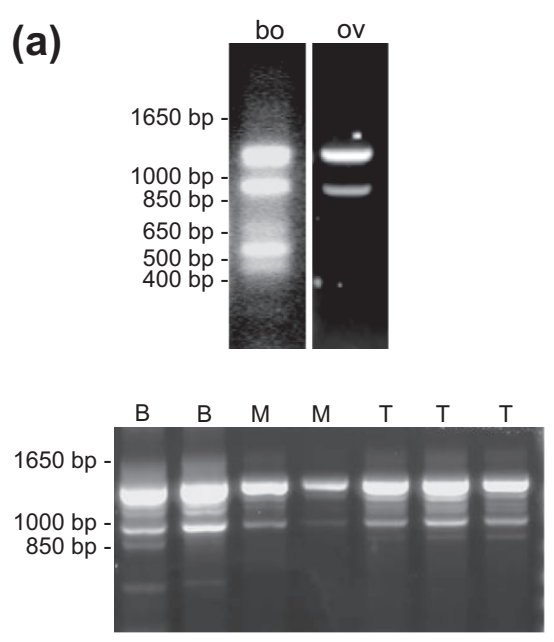

(b)

MR1 Splice Variants - bovine

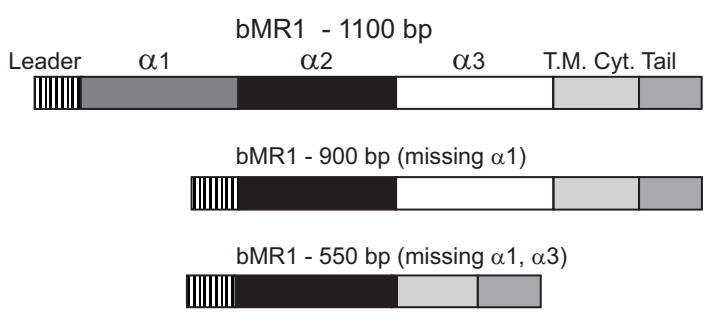

MR1 Splice Variants - ovine

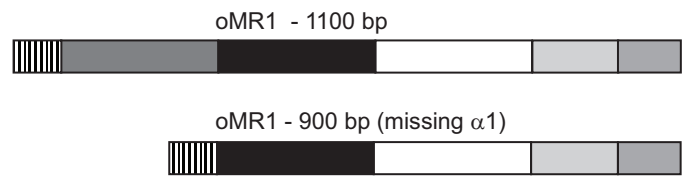

Figure 2. Detection of MR1 splice variants. (a) Agarose gel showing bands representing different splice variants of MR1 detected via PCR on cDNA from bovine and ovine PBMC (upper panel) and a variety of ovine Theileria-infected cell-lines (lower panel): two B-cell lines (B), two monocyte lines (M), and three T-cell lines (T). (b) Structure of MR1 splice variants detected in bovine and ovine PBMC. The $\alpha 2$ region is preserved in all variants identified.

from bovine PBMC. Sequencing of 8 clones from this PCR product revealed sequences that were identical or highly similar to the previously described bovine MAIT cell TCR sequence [18]. The sequences included two variants with single amino acid substitutions (CVVMDGNYQWI and CVVIDGNYQWI) at position 4 in the $\mathrm{CDR} 3$ region. Analysis of a further $7 \mathrm{cDNA}$ clones obtained from PBMC of a second animal identified 3 MAIT TCR $\alpha$ chain sequences. The remaining 4 clones also contained the MAIT $\mathrm{V} \alpha$ segment, but they had different CDR3 and $\mathrm{J}$ gene sequences.

Ovine MAIT TCR $\alpha$ sequences were obtained from PBMC by PCR using a primer located in the $5^{\prime}$ untranslated region of bovine sequences containing the MAIT V $\alpha$, along with a reverse primer in the ovine TCR $\alpha$ constant region. Sequencing of 7 cloned cDNA from the PCR product identified 2 clones with 

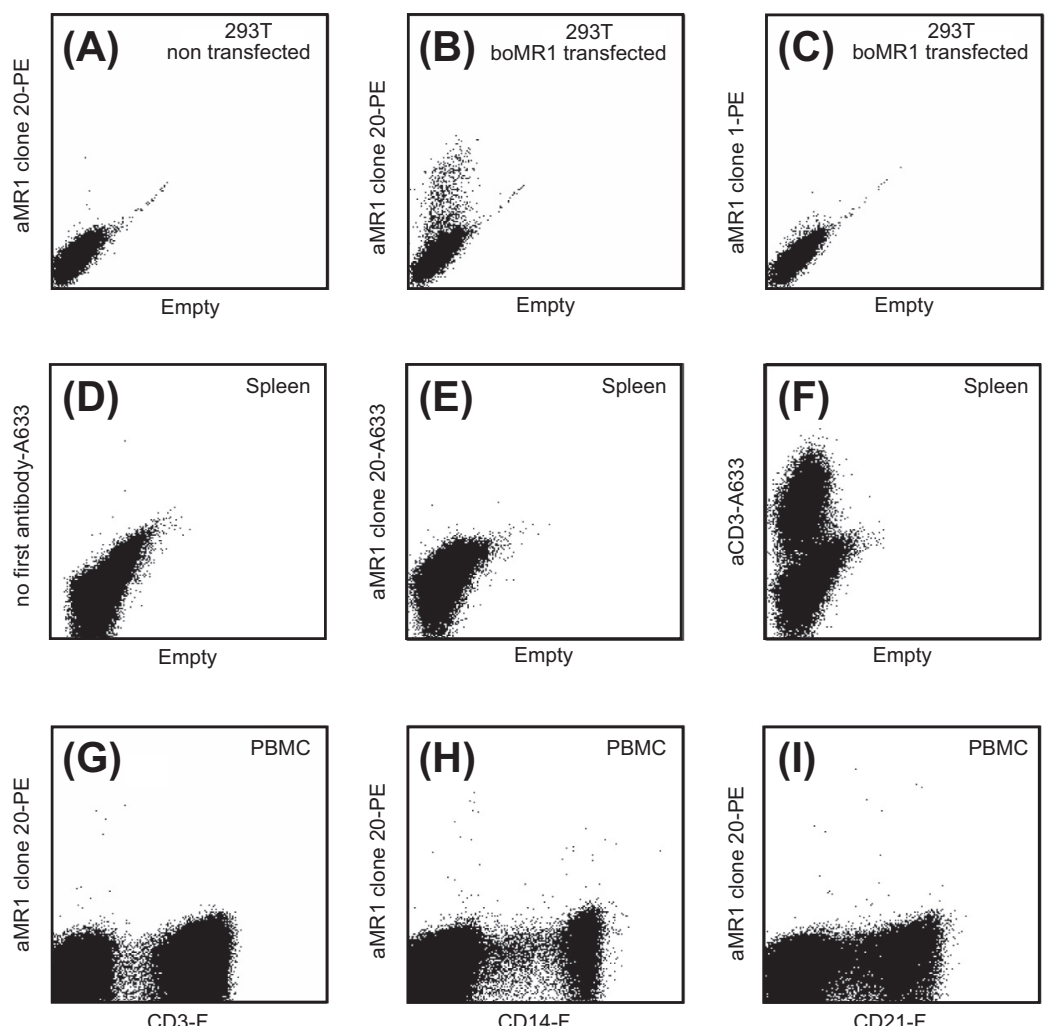

Figure 3. Flow cytometric detection of MR1. 293T cells were transfected with boMR1 or mock-transfected and stained with a panel of anti human MR1 antibodies. Monoclonal antibody clone 20 stained transfected cells (B), but not mock-transfected cells (A). Isotype-matched clone 1 did not stain transfected cells (C). Ex vivo cell suspensions from spleen (D-F), thymus, ileum, colon and prescapular lymph node (not shown) were stained with anti MR1 clone 20 or with anti bovine CD3. Ex vivo bovine PBMC were double stained with anti MR1 clone 20 and anti bovine CD3, CD14 or CD21 (G-I). All stainings were performed using a protocol that stains intracellular plus cell surface molecules.

identical sequences that closely matched the full-length bovine MAIT sequence. The V $\alpha$ sequence in these cDNA showed $96 \%$ similarity with bovine MAIT $\mathrm{V} \alpha$. The $\mathrm{J}$ region of these clones showed three single nucleotide differences compared with bovine, all resulting in coding changes; two of these differences were in the CDR3 and resulted in amino acid substitutions at positions 10 and 11 of the predicted CDR3 sequence (CAVMDGNYRLI rather than CAVMDGNYQWI). Based on the high level of sequence similarity with the bovine MAIT $\mathrm{TCR} \alpha$, we concluded that these sequences represented ovine MAIT TCR $\alpha$. The remaining 5 cDNA obtained from the original PCR contained the same $\mathrm{V}$ gene but had different CDR3 region sequences including different $\mathrm{J}$ segments. Thus, in both species, a substantial proportion of $\mathrm{T}$ cells expressing the MAIT V segment orthologue have the invariant MAIT TCR $\alpha$ chain.

Alignment of the bovine, ovine, murine and human MAIT cell V $\alpha$ segment and CDR3 $\alpha$ shows that the CDR1 and CDR2 region sequences in all 4 species are identical except for one amino acid substitution in the murine 


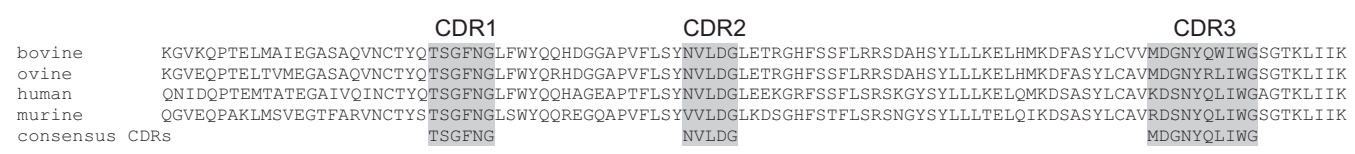

Figure 4. Comparison of MAIT TCRV $\alpha$ chain CDR. Alignment of the predicted amino acid sequences of the $\mathrm{V}$ segment and CDR regions (grey boxes) of the MAIT TCR $\alpha$ chain from cattle, sheep, humans and mouse.

CDR2, and that their CDR3 regions differ by only one or two amino acids (Fig. 4).

\subsection{Relative abundance of MAIT cells in the total $\alpha \beta T$ cell pool of different tissues}

We compared the relative transcription of the MAIT TCR $\alpha$ chain to the total pool of $\alpha$ chains in cell suspensions from peripheral blood, peripheral lymph node, ileum, colon, thymus and spleen, as well as mesenteric lymph node in some experiments. For this purpose, we used two qPCR reactions; one to estimate the number of constant $\alpha$ chains per tissue sample and the second to estimate the number of MAIT TCR $\alpha$ chains in the same sample. One of the aforementioned full-length (bovine or ovine, as appropriate) MAIT cell TCR $\alpha$ chain clones was used to generate a standard curve for each reaction. All experimental reactions for standards and samples were performed in triplicate. Initial experiments were performed upon two 3-month old calves and two 6-month old sheep, and for each animal the qPCR experiment was performed on 2 or 3 independent occasions. Results are presented in Figure 5. The estimated percentages of MAIT cells within each organ, based on relative abundance of the specific TCR $\alpha$ transcripts were similar between the pairs of calves and sheep, as well as for each individual experimental run. In calves the highest percentages of MAIT cells (roughly 1-3\%) were found in spleen and ileum. All other tissues showed comparatively lower MAIT cell levels. The two sheep showed the highest percentages of MAIT cells $(0.3-0.5 \%)$ in prescapular lymph node, spleen and ileum, while the levels in thymus, colon and PBMC were all $<0.1 \%$. Comparison of the two species shows notably higher percentages in general in cattle. With the exception of the prescapular lymph node, the tissue preference of MAIT cells is similar when the two different species are compared: MAIT cells are more abundant in spleen and ileum, and less so in thymus, colon and PBMC.

\subsection{Effect of age upon the prevalence and distribution of MAIT cells}

To determine the effect of age upon MAIT cell prevalence and distribution we used the above qPCR reaction to study the relative transcription of MAIT TCR $\alpha$ chain to the total pool of $\alpha$ chains in tissues from calves of different ages within the first 3-months of life. Three neonatal calves, three 3 -week old calves, and three 3-month old calves were used. Blood samples from the 3-week old calves were also collected on the day of birth to allow animalspecific comparison of MAIT cell levels within peripheral blood. Results are presented in Figure 6. Neonatal animals showed consistently low levels of MAIT cells throughout all tissues tested, and statistical analysis of experimental data shows a highly significant increase in MAIT cell abundance in 3-week $(p<0.001)$ and 3-month $(p=0.044)$ animals when compared to neonates. In particular, spleen and mesenteric lymph node show marked increases in MAIT expression by 3-weeks of age, while ileum, colon, PBMC and peripheral lymph node showed less pronounced increases. The increased MAIT cell abundance for each organ appears to be maintained at this level in the 3-month old animals. By contrast, MAIT levels remained minimal within the thymus irrespective of age. Statistical analysis of these data showed MAIT cell levels in the spleen to be highly significantly raised when compared to 

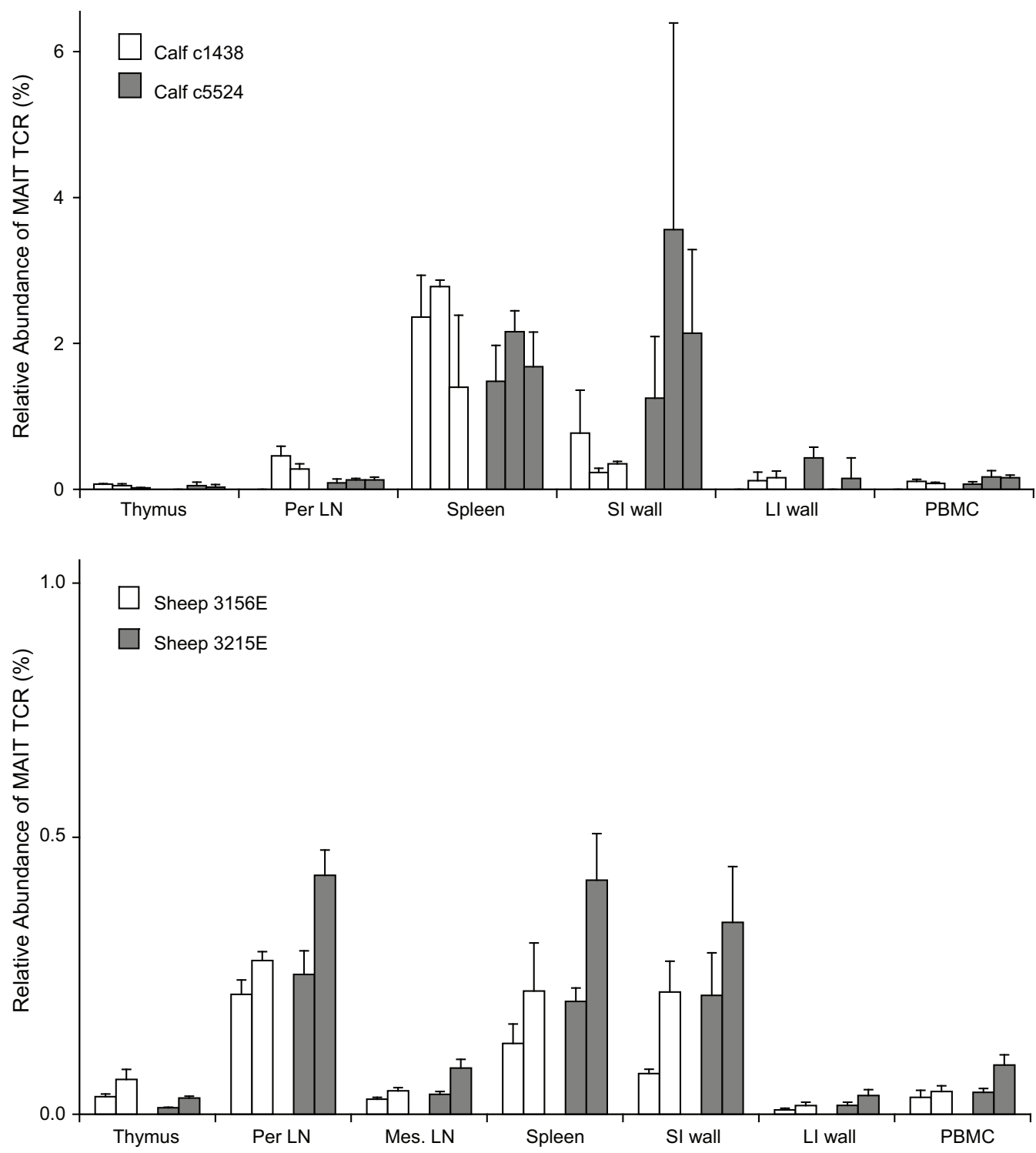

Figure 5. Estimated numbers of MAIT cells within the T cell populations in tissues of cattle and sheep. Samples of cDNA prepared from bovine and ovine tissues were analysed by qPCR to determine the number of MAIT cell TCR $\alpha$ chain transcripts and the number of $\alpha$ chain constant segment transcripts present. Numbers of transcripts were calculated by comparison with a standard curve that was generated using a cloned full-length MAIT cell $\alpha$ chain. Results are presented as quantities of MAIT TCR $\alpha$ chain expressed as a percentage of total $\alpha$ chain transcripts. Each experiment was performed three times on the same tissues from each animal. Per LN: peripheral lymph node; Mes LN: mesenteric lymph node; SI wall: small intestinal wall; LI wall: large intestinal wall. 


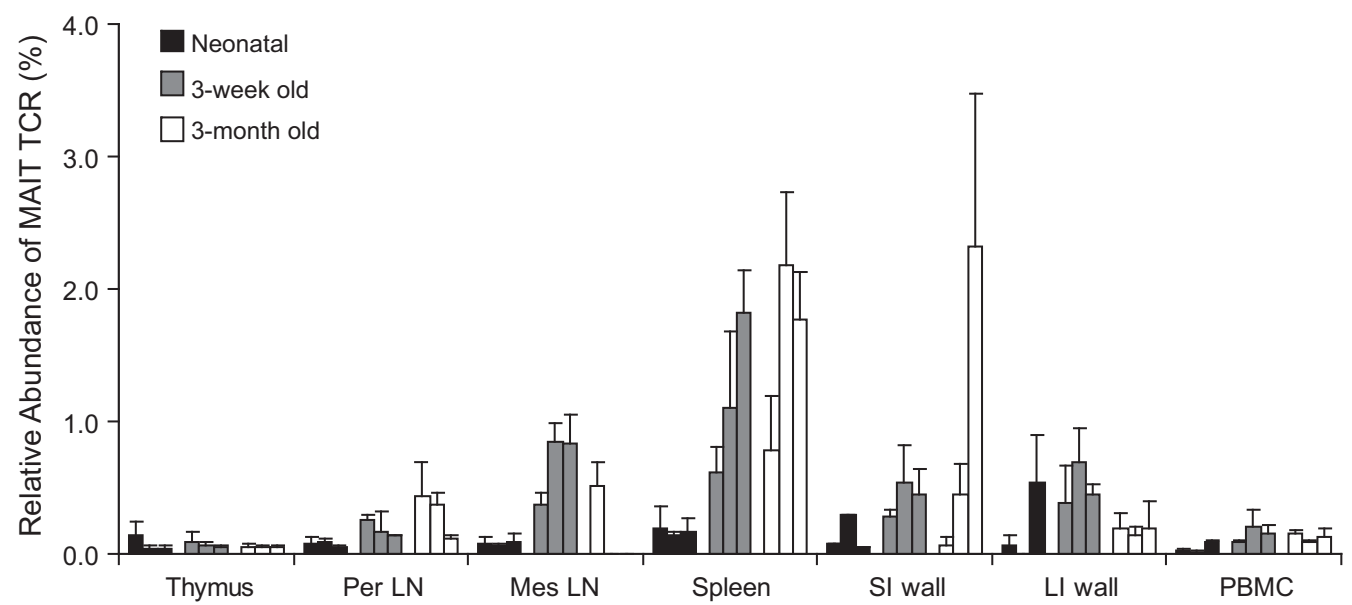

Figure 6. Effect of age on MAIT cell abundance. Abundance of MAIT cell TCR $\alpha$ chain transcripts per constant region transcripts was measured by qPCR in tissues from calves of three different age groups: neonatal, 3-week old and 3-month old. Results are presented as quantities of MAIT TCR $\alpha$ expressed as a percentage of total $\alpha$ chain transcripts, and represent the mean of three identical reactions run for each tissue sample. Mesenteric lymph nodes were not assessed in two out of three 3-month old. Per LN: peripheral lymph node; Mes LN: mesenteric lymph node; SI wall: small intestinal wall; LI wall: large intestinal wall.

thymus $(p<0.001)$, PBMC $(p<0.001)$ and peripheral lymph node $(p=0.001)$. Percentages of MAIT cells in mesenteric lymph node, ileum and colon were highly significantly increased in comparison to thymus $(p<0.001, p<0.001$, $p=0.002)$ and significantly increased in comparison to PBMC $(p=0.016, p=0.015$, $p=0.041)$.

\section{DISCUSSION}

Human and murine MAIT cells express an invariant TCR $\alpha$ chain incorporating the orthologous $\mathrm{V}$ and $\mathrm{J}$ gene segments and near identical CDR3 region sequences. MAIT cells are thought to be restricted by the MHC class I-like molecule MR1 because the development of MAIT cells in mice is dependent on the presence of the gene for MR1, and MAIT cell hybridomas recognize MR1 in vitro. The high level of homology between the murine, human, bovine, and ovine MR1 and MAIT TCR $\alpha$ chain sequence that we describe here confirms the evolutionary conservation of the MR1/MAIT system between these species.
We have furthermore demonstrated that, as in humans and mice, MR1 protein expression in ruminants is not detectable by flow cytometry in cells harvested ex vivo. In the current study, a monoclonal antibody raised against human MR1 was used that, based on analysis of cells transfected with bovine MR1 cDNA, was shown to cross-react with the bovine protein. Only intracellular expression of MR1 was detected on transfectant cells, and the antibody did not give any intracellular or surface staining of bovine cells harvested ex vivo. This result may reflect absence of MR1 protein expression in vivo, which may be strictly regulated, but it is also possible that there is MR1 expression in vivo at a level below our detection limit. Low sensitivity of our staining method may be due to lower affinity of the antibody for bovine MR1 compared to human MR1. This apparent lack of detectable protein expression in vivo suggests that in addition to being structurally highly homologous to the murine and human, the MR1/MAIT cell system is also functionally highly conserved.

A remarkable feature of the analysis of MR1 transcripts in both cattle and sheep was the 
detection of an alternatively spliced variant lacking the $\alpha 1$ exon, which was abundant both in PBMC and in several bovine cell lines (Fig. 2). A less abundant transcript, which lacked both the $\alpha 1$ and $\alpha 3$ domains, was also identified in cattle. Both variants retained the original reading frame and would be predicted to encode polypeptides with intact transmembrane and cytoplasmic domains. A number of alternatively spliced variants of MR1 have also been described in humans and mice [14]. Although many of the splice variants in the mouse also had disrupted $\alpha 1$ domains these mouse variants retained a small part of the $\alpha 1$ exon. By contrast, the human MR1 variants most frequently exhibited in-frame truncation of the $\alpha 3$ exon.

Quantitative PCR analysis using primers specific for MAIT TCR V $\alpha$ and CDR3, together with an independent PCR for total TCR $\alpha$ chain transcripts, was used in the current study to estimate the relative abundance of MAIT cells in different tissues. This provided estimates of MAIT TCR usage in $1-2 \%$ of all $\alpha \beta$ T cells in those tissues in which transcripts were most abundant. These percentages are far in excess of what can be expected for sequences generated by random rearrangement. Very low levels of MAIT transcripts were detected in the thymuses of calves of different ages and in all tissues of neonatal calves. A marked and statistically significant increase in transcripts in certain peripheral tissues was observed in 3 -week old animals. These findings are consistent with observations in mice indicating that extrathymic expansion of this population occurs post-natally. Initial studies failed to detect MAIT cells in human or murine thymus using a highly-sensitive RT-PCR [18]. More recently, Martin et al. [9] have demonstrated that MAIT cells are positively selected within the thymus in an MR1-dependent manner but undergo expansion in the periphery. The latter has been shown to be dependent on the presence of B-cells and an intact intestinal bacterial flora.

In comparison to thymus and PBMC, significantly increased levels of MAIT TCR $\alpha$ chain transcripts were detectable in samples of small intestine and mesenteric lymph nodes of 3-week and 3-month old calves. However, transcripts were found most consistently and most abundantly in the spleen. This is the first evidence that MAIT cells represent a prominent $\mathrm{T}$ cell population in the spleen, as there are no comparable data on MAIT cell TCR expression in human or murine spleen. Our findings raise questions concerning the role of the gut as a site of expansion of these cells. The finding that expansion of the MAIT cell population in mice is dependent on gut flora has prompted the suggestion that a microbe-derived MR1-presented ligand may cause local MAIT cell expansion [20]. Alternatively, the primary role of the gut flora may be to activate and expand B cell populations, which then migrate to other systemic lymphoid tissues including the spleen, where they can stimulate expansion of MAIT cell populations. In this model, the abundance of MAIT cells in the bovine spleen may reflect the fact that this tissue is relatively rich in B cells and has numerous active germinal centers [10].

Acknowledgements. We would like to thank Dr S. Gilfillan and Dr M. Cella, Department of Pathology and Immunology, Washington University School of Medicine, St. Louis for providing anti MR1 antibodies. I. Van Rhijn is supported by a Meervoud subsidy of the NWO (Nederlandse Organisatie voor Wetenschappelijk Onderzoek). N. Goldfinch and T. Connelley were supported by a grant awarded through the VTRI initiative by the UK Department for Food and Rural Affairs and the Scottish Higher Education Funding Council.

\section{REFERENCES}

[1] Goddeeris B.M., Morrison W.I., Techniques for the generation, cloning, and characterization of bovine cytotoxic T cells specific for the protozoan Theileria parva, J. Tissue Cult. Methods (1988) 11:101-110.

[2] Hansen T.H., Huang S., Arnold P.L., Fremont D.H., Patterns of nonclassical MHC antigen presentation, Nat. Immunol. (2007) 8:563-568.

[3] Hashimoto K., Hirai M., Kurosawa Y., A gene outside the human MHC related to classical HLA class I genes, Science (1995) 269:693-695.

[4] Howard C.J., Sopp P., Parsons K.R., Bembridge G.P., Hall G., A new bovine leukocyte antigen cluster comprising two monoclonal antibodies, CC43 and CC118, possibly related to CD1, Vet. Immunol. Immunopathol. (1993) 39:69-76. 
[5] Huang S., Gilfillan S., Cella M., Miley M.J., Lantz O., Lybarger L., et al., Evidence for MR1 antigen presentation to mucosal-associated invariant T cells, J. Biol. Chem. (2005) 280:21183-21193.

[6] Huang S., Gilfillan S., Kim S., Thompson B., Wang X., Sant A.J., et al., MR1 uses an endocytic pathway to activate mucosal-associated invariant T cells, J. Exp. Med. (2008) 205:1201-1211.

[7] Huang S., Martin E., Kim S., Yu L., Soudais C., Fremont D.H., et al., MR1 antigen presentation to mucosal-associated invariant T cells was highly conserved in evolution, Proc. Natl. Acad. Sci. USA (2009) 106:8290-8295.

[8] Mackay C.R., Maddox J.F., Gogolin-Ewens K.J., Brandon M.R., Characterization of two sheep lymphocyte differentiation antigens, SBU-T1 and SBUT6, Immunology (1985) 55:729-737.

[9] Martin E., Treiner E., Duban L., Guerri L., Laude H., Toly C., et al., Stepwise development of MAIT cells in mouse and human, PLoS Biol. (2009) 7:e54.

[10] Masake R.A., Morrison W.I., Evaluation of the structural and functional changes in the lymphoid organs of Boran cattle infected with Trypanosoma vivax, Am. J. Vet. Res. (1981) 42:1738-1746.

[11] Miley M.J., Truscott S.M., Yu Y.Y., Gilfillan S., Fremont D.H., Hansen T.H., Lybarger L., Biochemical features of the MHC-related protein 1 consistent with an immunological function, J. Immunol. (2003) 170:6090-6098.

[12] Okamoto N., Kanie O., Huang Y.Y., Fujii R., Watanabe H., Shimamura M., Synthetic alphamannosyl ceramide as a potent stimulant for an NKT cell repertoire bearing the invariant Valpha19-Jalpha26 TCR alpha chain, Chem. Biol. (2005) 12:677-683.

[13] Reinink P., Van Rhijn I., The bovine T cell receptor alpha/delta locus contains over $400 \mathrm{~V}$ genes and encodes $\mathrm{V}$ genes without CDR2, Immunogenetics (2009) 61:541-549.

[14] Riegert P., Wanner V., Bahram S., Genomics, isoforms, expression, and phylogeny of the MHC class I-related MR1 gene, J. Immunol. (1998) 161:40664077.

[15] Shiina T., Ando A., Suto Y., Kasai F., Shigenari A., Takishima N., et al., Genomic anatomy of a premier major histocompatibility complex paralogous region on chromosome 1q21-q22, Genome Res. (2001) 11:789-802.

[16] Shimamura M., Huang Y.Y., Okamoto N., Watanabe Y., Murakami Y., Kinoshita T., et al., Glycolipids with nonreducing end alpha-mannosyl residues that have the potential to activate invariant Valpha19 NKT cells, Febs J. (2007) 274:2921-2932.

[17] Shimamura M., Huang Y.Y., Kobayashi M., Goji H., Altered production of immunoregulatory cytokines by invariant Valpha19 TCR-bearing cells dependent on the duration and intensity of TCR engagement, Int. Immunol. (2009) 21:179-185.

[18] Tilloy F., Treiner E., Park S.H., Garcia C., Lemonnier F., de la Salle H., et al., An invariant T cell receptor alpha chain defines a novel TAP-independent major histocompatibility complex class Ib-restricted alpha/beta T cell subpopulation in mammals, J. Exp. Med. (1999) 189:1907-1921.

[19] Treiner E., Duban L., Bahram S., Radosavljevic M., Wanner V., Tilloy F., et al., Selection of evolutionarily conserved mucosal-associated invariant T cells by MR1, Nature (2003) 422:164-169.

[20] Treiner E., Duban L., Moura I.C., Hansen T., Gilfillan S., Lantz O., Mucosal-associated invariant T (MAIT) cells: an evolutionarily conserved $\mathrm{T}$ cell subset, Microbes Infect. (2005) 7:552-559.

[21] Van Rhijn I., Koets A.P., Im J.S., Piebes D., Reddington F., Besra G.S., et al., The bovine CD1 family contains group $1 \mathrm{CD} 1$ proteins, but no functional CD1d, J. Immunol. (2006) 176:4888-4893.

[22] Yamaguchi H., Hirai M., Kurosawa Y., Hashimoto K., A highly conserved major histocompatibility complex class I-related gene in mammals, Biochem. Biophys. Res. Commun. (1997) 238: 697-702.

[23] Yamaguchi H., Kurosawa Y., Hashimoto K., Expanded genomic organization of conserved mammalian MHC class I-related genes, human MR1 and its murine ortholog, Biochem. Biophys. Res. Commun. (1998) 250:558-564.

[24] Yamaguchi H., Hashimoto K., Association of MR1 protein, an MHC class I-related molecule, with beta(2)-microglobulin, Biochem. Biophys. Res. Commun. (2002) 290:722-729. 